\title{
The nucleus of the nearby galaxy IC 342
}

\author{
A. Schulz ${ }^{1,3}$, R. Güsten ${ }^{2}$, B. Köster ${ }^{4,5}$, and D. Krause ${ }^{4,6}$ \\ 1 Institut f. Physik \& ihre Didaktik, Universität zu Köln, Gronewaldstr. 2, 50931 Köln, Germany \\ 2 Max-Planck-Institut f. Radioastronomie, Auf dem Hügel 69, 53121 Bonn, Germany \\ 3 Institut f. Astrophysik \& Extraterr. Forschung, Universität Bonn, Auf dem Hügel 71, 53121 Bonn, Germany \\ 4 I. Physikalisches Institut, Universität zu Köln, Universitätsstr. 17, 50937 Köln, Germany \\ 5 Pricewaterhouse Coopers, Hohenzollernring 21-23, 50672 Köln, Germany \\ ${ }^{6}$ Pixelpark, Friesenplatz 25, 50672 Köln, Germany
}

Received 7 July 2000 / Accepted 4 January 2001

\begin{abstract}
To study the different components of the molecular gas in the nuclear region of the nearby spiral galaxy IC $342,{ }^{12} \mathrm{CO}(3-2),{ }^{13} \mathrm{CO}(3-2)$ and $(2-1)$, as well as $\mathrm{HCN}(1-0)$ through (4-3) observations are presented and analyzed in conjunction with a variety of line and continuum emission data of the literature. We find several giant molecular clouds embedded in a medium with lower column and volume density. The gas shows strong density and temperature gradients with a very clumpy substructure; the lower-density gas is rather warm $(\leq 50 \mathrm{~K})$ and the temperature decreases with increasing density. While LVG calculations are contradicting our observations, we are able to explain all $\mathrm{CO}$ and [CII] line data by applying a model of photon dominated regions (PDR); for the gas seen in these lines we find no evidence for an additional dominant heating mechanism. On the other hand, for our HCN observations tracing the dense gas clumps $\left(n\left(\mathrm{H}_{2}\right) \approx 10^{6} \mathrm{~cm}^{-3}\right)$ where the influence of UV photons is expected to be less important than for less dense gas seen in $\mathrm{CO}\left(n\left(\mathrm{H}_{2}\right) \approx 10^{4} \mathrm{~cm}^{-3}\right)$, an LVG model approach should be applicable and, in fact, yields results which fit well the data and support the outlined scenario. For this gas component which is rather cool $\left(T_{\mathrm{k}} \leq 30 \mathrm{~K}\right)$, heating processes involving photoelectrons should be unimportant and other mechanisms like turbulent energy dissipation or cosmic rays should be considered. The structure of the nucleus of IC 342 with several giant molecular clouds with sizes of $\leq 20$ to 50 pc and masses of order $10^{6} M_{\odot}$ partly associated with H II regions and embedded into a lower-density interstellar medium shows striking similarities in terms of cloud distribution and their physical behaviour with our Galactic Centre region.
\end{abstract}

Key words. galaxies: individual: IC 342 - galaxies: galactic nuclei - galaxies: spiral - galaxies: interstellar matter - radio lines: molecular

\section{Introduction}

Adopting a distance for IC 342 of $1.8 \mathrm{Mpc}$ ( $1^{\prime \prime}$ corresponds to $8.7 \mathrm{pc}$ ) instead of $4.5 \mathrm{Mpc}$ (see McCall 1989), it can be suspected that the nucleus of the nearby spiral galaxy IC 342 (type Scd, H I diameter $\sim 50 \mathrm{kpc}$ ) may show some general similarities to our own Galactic Centre (see Downes et al. 1992 and references therein). For example, giant molecular clouds are found in its central region of similar size to those of the Galactic Centre like Sgr A and Sgr B2, and the total near infrared and far infrared luminosities of the inner $400 \mathrm{pc}$ are comparable to the equivalent region of our Galaxy.

Its proximity and its almost face-on orientation towards the observer provides a unique possibility to study the nucleus of a nearby spiral galaxy from a very favorable viewing angle $\left(i=25^{\circ}\right)$ without interference

Send offprint requests to: A. Schulz, e-mail: p341sch@mpifr-bonn.mpg.de from the disc: it is small enough to observe its real morphology with only little confusion due to different velocity components in the beam, but it is large enough to allow a kinematic analysis. All this is reflected in the variety of recent investigations of the neutral interstellar matter up to very high angular resolution $\left(\geq 2^{\prime \prime}\right)$.

Molecular line emission from IC 342 is quite concentrated towards the centre, and molecular line maps represent the inner kpc. Close inspection of the high-resolution maps in various molecular lines show interesting coincidences and differences: HCN (1-0) interferometer data $\left(2.7^{\prime \prime}\right.$ resol.; Downes et al. 1992) revealed 5 dense clouds of 10 to $50 \mathrm{pc}$ size along the central mini-spiral. ${ }^{12} \mathrm{CO}(1-$ 0 ) emission of Ishizuki et al. (1990, 2.4" resol.) and Meier et al. (2000, 4.5" res.) - which is usually interpreted to trace predominantly intercloud material - is very closely correlated to the $\mathrm{HCN}$ emission, whereas the map of ${ }^{13} \mathrm{CO}(1-0)$ emission ( $5^{\prime \prime}$ resol.) by Turner \& Hurt (1992) does not show the prominent HCN cloud " $\mathrm{B}$ " which is 
stated to be correlated to strong HII radio continuum emission. This picture gets even more complex regarding the $\mathrm{NH}_{3}$ map (5" resol.) of Ho et al. (1990) where some of the $\mathrm{HCN}$ clouds are well identifiable but some are missing.

Several investigations have shown that large differences in excitation conditions occur on small scales (see Downes et al. 1992; Güsten et al. 1993; Harris et al. 1992; Wall \& Jaffe 1990; Eckart et al. 1990). Attempts to model the observed line ratios by two or more emitting gas components (Downes et al.; Güsten et al.) suggest a component of small dense warm clouds (a few to $10 \mathrm{pc}, \geq 10^{4} \mathrm{~cm}^{-3}$, $\sim 50 \mathrm{~K})$ and at least one of less dense partly cooler interclump and spiral arm material. In order to derive possibly unique sets of physical parameters for the various gas components, it was particularly disadvantageous up to now that for HCN and CS, respectively, only one transition was observed with sufficiently high angular resolution.

It is the aim of this study to confine the properties of the different gas components resolving them spatially. As a tracer of warm gas of intermediate density, ${ }^{12} \mathrm{CO}$ and ${ }^{13} \mathrm{CO}(3-2)$ is well suited. To extend this analysis to very high density material, additionally several lines of $\mathrm{HCN}$ were measured covering a large range of excitation conditions (i.e. critical densities). Since the highest possible angular resolution is essential for this task, the IRAM $30 \mathrm{~m}$-telescope (MRT) is the only instrument at this time where this research is possible.

\section{Observations}

The observations were performed during various winter seasons from Jan. 1993 to Feb. 1998 at the IRAM 30 mtelescope (MRT) in the beam switching mode using the chopping secondary mirror with a throw of $200^{\prime \prime}$ in azimuth at a switching frequency of $0.5 \mathrm{~Hz}$. The integration time for a single measurement was typically $10^{\mathrm{min}}$; each position was repeatedly measured obtaining total integration times of up to 4 hours per position.

With molecular cloud sizes of $\leq 50 \mathrm{pc}\left(\sim 6^{\prime \prime}\right)$, a very good telescope pointing is essential; this was carefully checked every 30 to $40^{\mathrm{min}}$ on the nearby source $\mathrm{W} 3(\mathrm{OH})$ using the $3 \mathrm{~mm}$ and $1 \mathrm{~mm}$ receivers; data with pointing offsets of more than $1.5^{\prime \prime}$ ( $\sim 10 \%$ of the data) were ignored. At the beginning of each observing run, the parallel alignment of all used receivers (at $3 \mathrm{~mm}, 2 \mathrm{~mm}, 1 \mathrm{~mm}$ and $0.8 \mathrm{~mm}$ ) was achieved by observing planets.

- ${ }^{12}$ CO (3-2): The receiver was the $345 \mathrm{GHz}$ quasioptical Schottky diode mixer of the Max-PlanckInstitut für Extraterrestrische Physik, Garching (see Rothermel et al. 1994) which was installed at the MRT for the winter season 1993. The receiver noise temperature at $345.8 \mathrm{GHz}$ was $100 \mathrm{~K}$ (DSB). Extensive calibration measurements on the planets and on the Moon yielded foreward and main beam efficiencies of 0.8 and 0.17 , respectively; the main beam size was determined to be $8.3^{\prime \prime}$ (FWHM). Zenith atmospheric transparency $\tau_{0}$ ranged from 0.1 to 1.70 positions;
- ${ }^{13}$ CO (3-2): In the following winter season 1994, the IRAM $345 \mathrm{GHz}$ SIS mixer was installed for the first time. Its noise temperature was $\leq 100 \mathrm{~K}$ (DSB, during the last session SSB) at $330.6 \mathrm{GHz}$. Calibration measurements yielded basically the same foreward and main beam efficiencies as the MPE $345 \mathrm{GHz}$ receiver in the previous season, the main beam size was again 8.3" (see Wild et al. 1994). $\tau_{0}$ ranged from excellent 0.1 up to 0.7. 25 observing positions;

- ${ }^{13} \mathbf{C O}(\mathbf{2}-\mathbf{1})$ : Since the ${ }^{13} \mathrm{CO}(2-1)$ data of Eckart et al. were observed on a $10^{\prime \prime}$ grid, this line $(220.4 \mathrm{GHz})$ was remeasured in parallel to our ${ }^{13} \mathrm{CO}(3-2)$ mapping using the $1 \mathrm{~mm}$ facility receiver (foreward and main beam efficiencies 0.9 and 0.45 , resp.); $\tau_{0}$ is about a factor of 3 lower than at $345 \mathrm{GHz}$. 25 observing positions.

- HCN (4-3): This transition at $354.505 \mathrm{GHz}$ was observed with the IRAM $345 \mathrm{GHz}$ SIS receiver at a few positions of maximum CO (3-2) and HCN (1-0) emission during the last season in Feb. 1998. The system performance was similar to that for ${ }^{13} \mathrm{CO}(3-2)$;

- HCN (3-2) and ${ }^{\mathbf{1 3}} \mathbf{H C N}$ (3-2): The HCN (3-2) line at $265.886 \mathrm{GHz}$ was observed during Jan. 1997 using the second IRAM $1 \mathrm{~mm}$ receiver which has at this frequency at its band limit a noise temperature as high as $400 \mathrm{~K}$. Also $\tau_{0}$ is slightly higher at this frequency than at $230 \mathrm{GHz}$. Foreward and main beam efficiencies were 0.85 and 0.32 , resp. at $265.9 \mathrm{GHz}$, beam size was $10^{\prime \prime} .5$ positions observed;

- HCN (2-1): This line was observed in parallel to $\mathrm{HCN}(3-2)$ using the IRAM $2 \mathrm{~mm}$ receiver at 177.236 GHz. Due to an atmospheric absorption band, $\tau_{0}$ is rather high in most cases (0.5 to 0.8$)$. System efficiencies were 0.9 and 0.50 , resp. Beam size: $14^{\prime \prime}$. Only 2 positions observed;

- $\mathbf{H C N}(\mathbf{1}-\mathbf{0})$ and $\mathbf{H}^{\mathbf{1 3}} \mathbf{C N}(\mathbf{1}-\mathbf{0})$ : The lowest HCN rotational transition at $88.6 \mathrm{GHz}$ was observed with the IRAM $3 \mathrm{~mm}$ receiver under usual performance conditions mainly for calibration purpose to compare with data by Downes et al. in parallel to $\mathrm{HCN}(3-2)$ for short integration times; during most of the $\mathrm{HCN}(3-2)$ observations, $\mathrm{H}^{13} \mathrm{CN}(1-0)$ at $86.342 \mathrm{GHz}$ was measured with a $27^{\prime \prime}$ beam and added together later on.

With an unfavorable ratio of main beam and error pattern at $345 \mathrm{GHz}$, it is obvious that one can observe only compact sources without having severe problems of beam smearing. In this respect, it is important that no prominent side lobes close to the main beam are found; the error beam consists of a very extended pedestal of low sensitivity. It is also obvious that for sources larger than the main beam a reasonable antenna temperature scale in order to compare our data to those of the literature is not simply given by the usually applied main beam brightness temperature $T_{\mathrm{mb}}$; it is sensible to apply an effective main beam brightness temperature $T_{\mathrm{eb}}$ which is corrected for the effective beam efficiency $\eta_{\mathrm{eb}}$ according to the source size (for details see Steppe et al. 1990). For example, for 


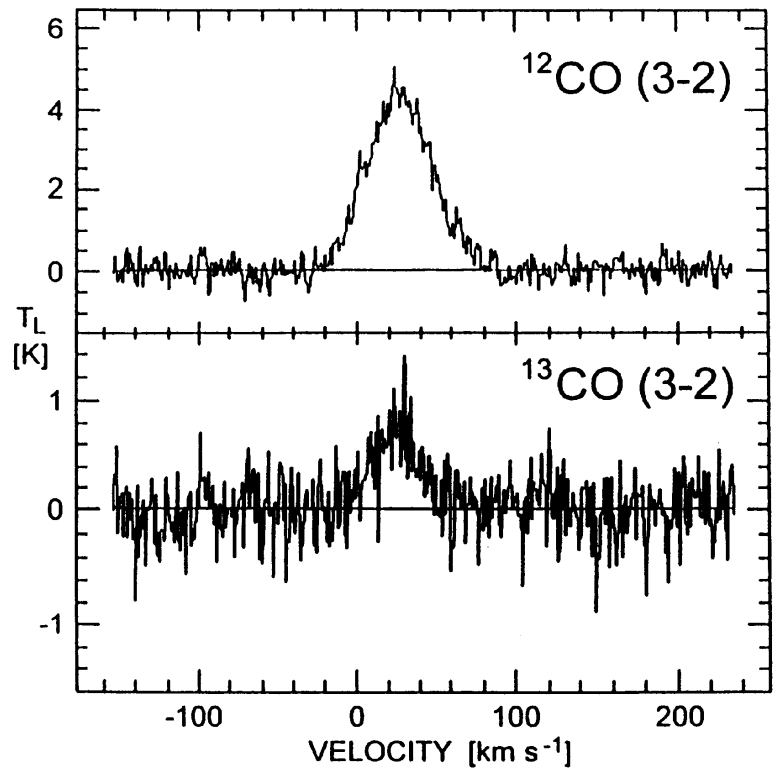

Fig. 1. Spectra of ${ }^{12} \mathrm{CO}(3-2)$ and ${ }^{13} \mathrm{CO}(3-2)$ (spect. resol. $\left.0.9 \mathrm{~km} \mathrm{~s}^{-1}\right)$ at the reference position of $\alpha(1950)=3 \mathrm{~h} 41 \mathrm{~m} 57.0 \mathrm{~s}$, $\delta(1950)=67^{\circ} 56^{\prime} 29^{\prime \prime}$ used throughout this paper (full velocity range, unsmoothed). The line temperature $T_{\mathrm{L}}$ is equal to $T_{\mathrm{eb}}$ discussed in the text

the IC $342{ }^{12} \mathrm{CO}(3-2)$ map $\eta_{\mathrm{eb}}$ was determined to be 0.21 . All other line intensities are expressed in $T_{\mathrm{mb}}$. The dependence of antenna gain on elevation angle was also included in the calibration.

We used the IRAM facility $1 \mathrm{MHz}$-backends (filter banks and autocorrelator).

\section{Results}

\subsection{The CO measurements}

Figure 1 presents the $\mathrm{CO}(3-2)$ and ${ }^{13} \mathrm{CO}(3-2)$ spectrum at the $(0,0)$ position of our map (the same reference position was also used by Eckart et al. 1990 and Downes et al. 1992). Figures 2, 3 and 4 depict the 70-point map of the integrated intensity of the ${ }^{12} \mathrm{CO}(3-2)$ and the 25-point maps of the ${ }^{13} \mathrm{CO}(3-2)$ and ${ }^{13} \mathrm{CO}(2-1)$ spectra, all measured on a $4^{\prime \prime}$ grid (full velocity range of -30 to $110 \mathrm{~km} \mathrm{~s}^{-1}$ ).

The general distribution of integrated intensity is similar to other high-resolution molecular line maps of IC 342: we observe the ridge of the mini-spiral centered at the position of the $2 \mu \mathrm{m}$ emission maximum of Becklin et al. (1980) with its arms trailing those observed in $\mathrm{H}_{\alpha}$ (see Turner \& Hurt 1992). The northern arm is slightly more intense than the southern one which can also be seen regarding the $(3-2)$ line peak antenna temperatures.

Our ${ }^{13} \mathrm{CO}(3-2)$ emission is even more concentrated to the central region of IC 342 (which may partly be an effect of the lower signal-to-noise ratio), but the structure of the map (i.e. the positions of peaks and the contrast within the map) is the same as in ${ }^{12} \mathrm{CO}$. Regarding its slightly lower angular resolution, the ${ }^{13} \mathrm{CO}(2-1)$ map also reveals this same structure. For the emission maxima we keep the

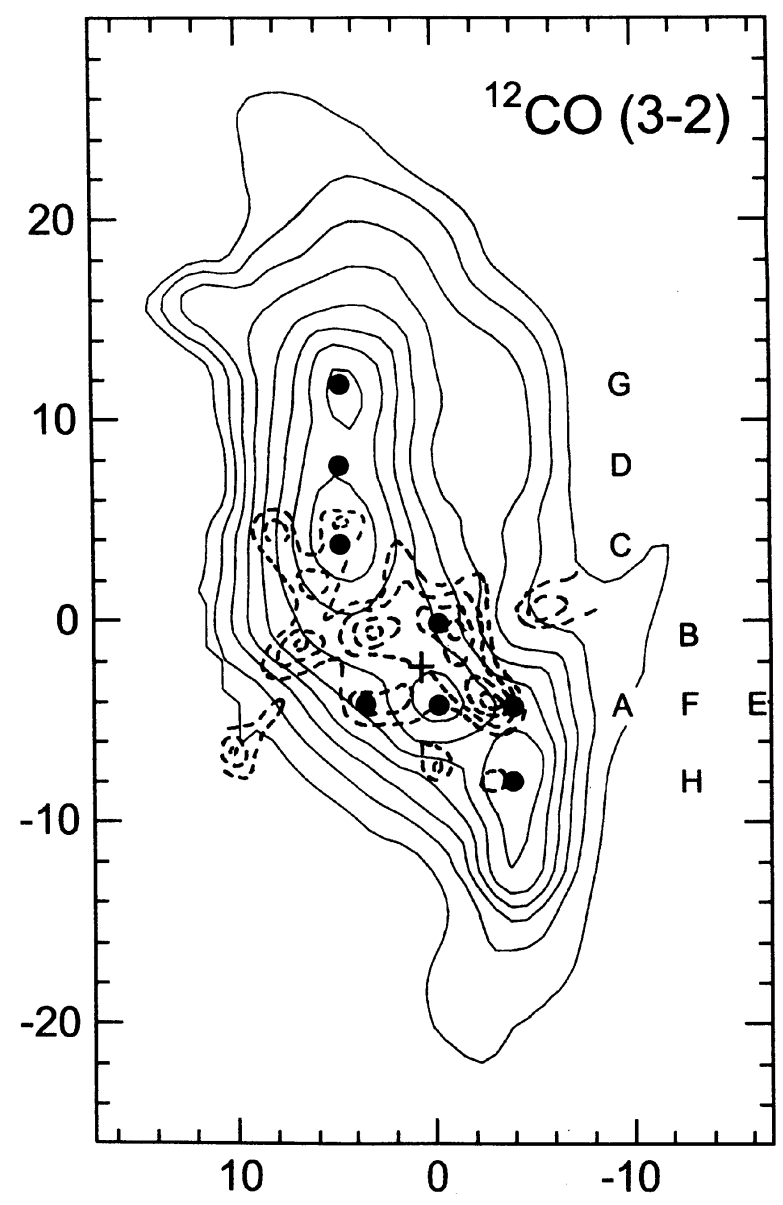

Fig. 2. Map of integrated ${ }^{12} \mathrm{CO}(3-2)$ line emission (vel. range -30 to $110 \mathrm{~km} \mathrm{~s}^{-1}$ in contours of 100 to $240 \mathrm{~K} \mathrm{~km} \mathrm{~s}^{-1}$ $\left(20 \mathrm{~K} \mathrm{~km} \mathrm{~s}^{-1}\right.$ increment). Offsets in $\operatorname{arcsec}((0,0)$-position as in Fig. 1). Dashed lines: $6 \mathrm{~cm}$ continuum emission (2" resol.) by Becklin et al. (1980) (their Fig. 1), the cross denotes their $2 \mu \mathrm{m}$ emisson maximum, the adopted centre of the galaxy. Thick dots mark cloud positions A-H (see Table 1)

notation of Downes et al. (1992) calling them "clouds" and extend it by cloud F, G and $\mathrm{H}$.

If we compare our CO (3-2) map with that of Steppe et al. (1990) obtained at the same telescope but with a Schottky diode receiver with a 10-times lower sensitivity than now, we see generally a fair agreement concerning calibration and intensity distribution; a major discrepancy appears only at the position east of cloud $\mathrm{C}$ which could possibly be caused by a pointing offset in the older map where much larger integration times had to be applied. Our recent spectra are of much higher S/N-quality.

\subsection{The HCN measurements}

Due to the weak intensities of the HCN lines it was not possible to fully map the nuclear region of IC 342 in any of the transitions, but we could take spectra at a few positions showing CO (3-2) maxima. For clump C, four HCN transitions are shown in Fig. 5. Table 3 contains all obtained line intensities scaled to a $10^{\prime \prime}$ beam (using $\mathrm{HCN}$ source sizes of Downes et al. 1992). 


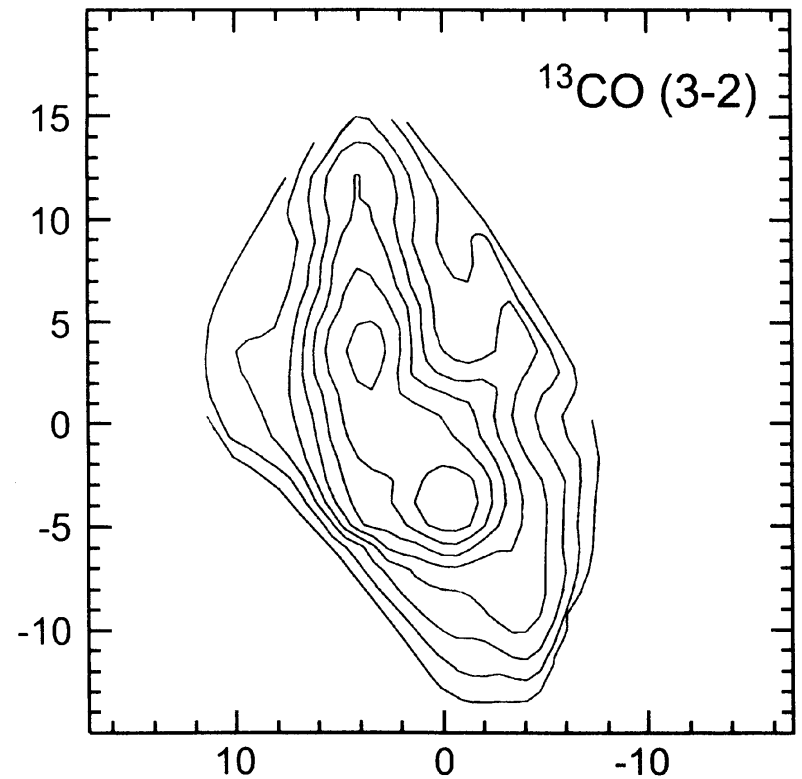

Fig. 3. Map of integrated ${ }^{13} \mathrm{CO}(3-2)$ line emission (positions and velocity range as in Fig. 2) in contours of 11 to $25 \mathrm{~K} \mathrm{~km} \mathrm{~s}^{-1}$ ( $2 \mathrm{~K} \mathrm{~km} \mathrm{~s}^{-1}$ increment)

First of all, obviously our HCN (1-0) spectrum at the $(0,0)$-position agrees with that of Downes et al. within $10 \%$ whereas there are large differences to the spectra of Rieu et al. (1992, 27" resol.) and Paglione et al. (1997, $20^{\prime \prime}$ resol.). On the other hand, our $\mathrm{H}^{13} \mathrm{CN}(1-0)$ spectrum shown in Fig. 5, despite its weak intensity, agrees with that of Paglione et al. within 20\%; our measured isotopic (1-0) line ratio is 24 .

Concerning HCN (3-2), our observations are in accordance with Paglione et al. within $20 \%$ regarding the largely different beams (within their 27" beam, Paglione et al. "see" at least 3 of the clumps). Concerning HCN (43 ), our spectrum ( $8.3^{\prime \prime}$ resol.) is by a factor of 2 less intense than that of Jackson et al. (1995, 20" resol.) at the comparable position.

Our limited observing time unfortunately did not allow to measure HCN (3-2) and (4-3) spectra at the positions of clumps $\mathrm{A}$ and $\mathrm{B}$.

\subsection{Alcohol in IC 342?}

We have also attempted to measure $\mathrm{H}^{13} \mathrm{CN}(3-2)$ with $12^{\prime \prime}$ resolution at the clump positions. The result was a spectral line of order $100 \mathrm{mK}$ for clumps $\mathrm{C}$ and $\mathrm{F}$ which is about the same as for the main isotopic line. Furthermore, the velocity offset of the 4 clump spectra contradicts the position-velocity relation in IC 342 observed in the other molecular lines: cloud $\mathrm{G}$ would be at the lowest and cloud $\mathrm{H}$ is at the highest velocity. Hence, this line can not be $\mathrm{H}^{13} \mathrm{CN}(3-2)$ emission but is arising from the image side band of the receiver; it is centered at $267.042 \mathrm{GHz}$ which is close to $267.045 \mathrm{GHz}$, the frequency of a pair of ethanol $\left(9_{8}-8_{8}\right)$ transitions. Although Millar et al. (1995) report a rather high ethanol abundance of $10^{-8}$ relative to hydrogen for the ultracompact galactic H II region G $34.3+0.15$,

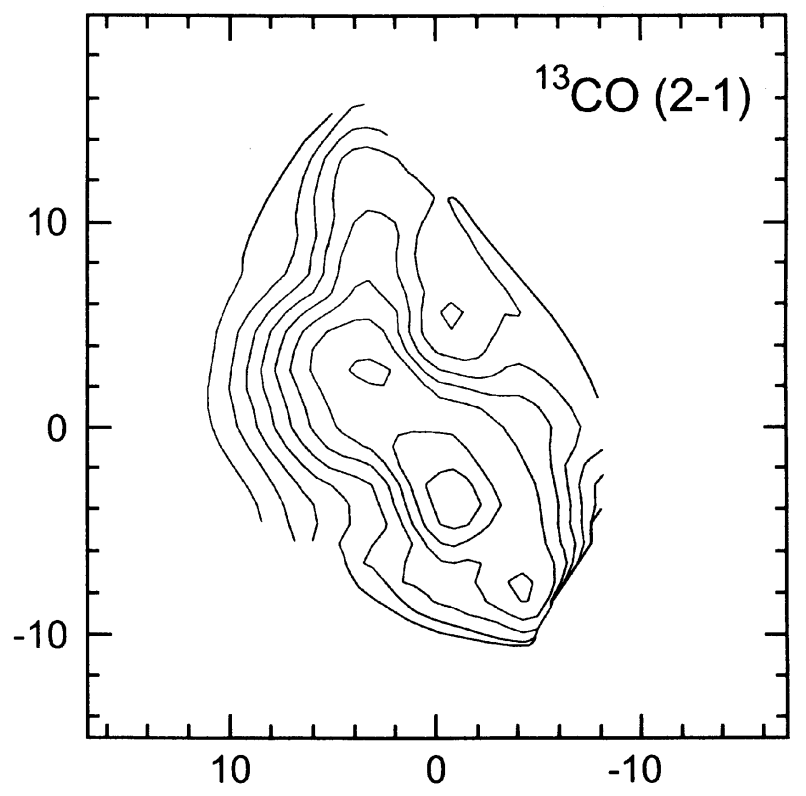

Fig. 4. Map of integrated ${ }^{13} \mathrm{CO}(2-1)$ line emission (positions and vel. range as in Fig. 2) in contours of 12 to $32.5 \mathrm{~K} \mathrm{~km} \mathrm{~s}^{-1}$ $\left(2.5 \mathrm{~K} \mathrm{~km} \mathrm{~s}^{-1}\right)$

in Sgr B2 this abundance is found to be a factor of order 10 lower (Nummelin et al. 2000). Therefore, due to the rareness of this molecule, this identification must appear to be extremely questionable. On the other hand, all other transitions within $\pm 30 \mathrm{MHz}$ listed in molecular line catalogues have energies of their lower state of several $100 \mathrm{~K}$ and are therefore even more unlikely candidates for extragalactic emission from an $\sim 70$ pc size area. This needs further investigation.

Within the observed line profiles we estimate an upper limit for the blended $\mathrm{H}^{13} \mathrm{CN}(3-2)$ emission of $10-20 \mathrm{mK}$ for clouds $\mathrm{C}$ and $\mathrm{F}$.

\section{Excitation analysis}

\subsection{CO lines}

\subsubsection{LVG radiative transfer models}

In a first approach to a physical interpretation, we attempted to model the molecular interstellar medium of the nucleus of IC 342 with a standard radiative transfer analysis using a single-component LVG (Large Velocity Gradient) line escape probability code to model the $\mathrm{CO}(3-2)$ and $(2-1)$ transitions $\left({ }^{12} \mathrm{CO}(2-1)\right.$ data from Eckart et al. 1990) at the different maxima of CO and HCN. As in the case of Güsten et al. (1993) for the centre position at $15^{\prime \prime}$ resolution, we failed to obtain a solution fitting all available CO lines. Güsten et al. also demonstrated that the ${ }^{13} \mathrm{CO}(3-2)$ line is a very sensitive probe for the physical gas conditions particularly in connection with (4-3) or higher excited CO lines.

This means that the observed line ratios require us to consider temperature (and possibly also density) gradients. Furthermore, even an LVG line escape probability 
Table 1. Source sizes

\begin{tabular}{lccccccccc}
\hline $\begin{array}{l}\text { "Cloud" } \\
\text { offset pos. }\end{array}$ & $\mathrm{G}$ & $\mathrm{D}$ & $\mathrm{C}$ & $\mathrm{F}$ & $\mathrm{A}$ & $\mathrm{B}$ & $\mathrm{E}$ & $\mathrm{H}$ \\
& $(4,12)$ & $(4,8)$ & $(4,4)$ & $(0,-4)$ & $(4,-4)$ & $(0,0)$ & $(-4,-4)$ & $(-4,-8)$ & \\
\hline & & & & & & & & & \\
$\mathrm{CO}(3-2)$ & 6 & $<6$ & 16 & 9 & - & - & $<6$ & $<6$ & $\left.\square^{\prime \prime} b\right)$ \\
$\mathrm{HCN}$ & $(\leq 4)$ & 7.7 & 8.3 & $(\leq 7)$ & 8.8 & 10.8 & 3.1 & $(\leq 4)$ & $\left.\square^{\prime \prime c}\right)$ \\
\hline
\end{tabular}

a) In arcsec from our $(0,0)$ position within our observing grid; to fit into our $4^{\prime \prime}$ grid of spectra, some positions are not exactly identical with Downes et al. (1992).

b) From this work.

c) From Downes et al. (1992); values in brackets are estimated.

model with 2 cloud components of different density and temperature permitted only a very limited range of resulting cloud parameters, being the same for regions close to the centre of IC 342 as also for regions further out along the mini-spiral which might be not realistic. Moreover, the CO line LVG models as well as a model with 3 cloud components by Downes et al. (1992) demand that the densest gas component is rather warm $(\geq 50 \mathrm{~K})$.

\subsubsection{PDR radiative transfer models}

In a second approach of interpretation, we applied a model of Photon Dominated Regions (PDR) (Köster et al. 1994) for the clouds within the central 400 pc of IC 342 .

This model calculates the thermal and chemical structure of a one-dimensional and plane-parallel PDR of finite size illuminated from both sides (model A of Köster et al.). The adopted chemistry includes a chemical network of 32 molecular species (244 reactions). Input parameters are the (fixed) gas density, the UV radiation strength, the total extent, the line width as well as the fractional abundances of $\mathrm{H}, \mathrm{He}, \mathrm{C}$ and $\mathrm{O}$ (incl. their abundant isotopes) and dust particles (scattering and absorption by grains). The main heating source is the interstellar UV radiation field between 6.2 and $13.6 \mathrm{eV}$ with a strength relative to the local field $\chi_{0}=210^{-4} \mathrm{erg}\left(\mathrm{cm}^{2} \mathrm{~s} \mathrm{sr}\right)^{-1}$ (Draine 1978). Heating is achieved via photoelectrons and - important at higher densities - collisional de-excitation of UV-pumped vibrationally excited $\mathrm{H}_{2}$; cooling is achieved via line emission of atomic fine structure lines and molecular lines.

Model results are abundances, absolute line intensities and column densities of the different atomic and molecular gas species as a function of visual extinction which is a measure of gas (and dust) column density. Additionally, the gas temperature is calculated throughout each "subcloud" (assumed velocity interval of $1.7 \mathrm{~km} \mathrm{~s}^{-1}$ ). The entire cloud then consists of a number of such sub-clouds at different velocities according to the number of velocity intervals within the total line width observed at the modelled position.

The assumption of plane-parallel geometry (observed face-on in our case) is not disturbing the results since the observed angular extent of the clumps defines their lateral extent. Furthermore, we emphasize that we prefer this rather simple model to minimize the number of free parameters which we find more appropriate in our approach modelling an entire galactic nuclear region where we observe averages of emission over linear dimensions of $\geq 20$ pc, rather than using a more sophisticated model with more free parameters for "fine tuning" which we can not yet confine observationally (e.g. Störzer et al. 2000); the latter would be definitely appropriate when analyzing nearby Galactic clouds with observed fine structure.

\subsubsection{PDR model results}

As also resulting from other models, within a rather large temperature range $\mathrm{CO}$ line ratios of different transitions (e.g. (3-2)/(2-1) ratios) are mainly determined by the gas density $n\left(\mathrm{H}_{2}\right)$; high density yields thermal excitation and line ratios close to 1 . But, different from other models, we consider gradients of the chemical and temperature structure; this is important since, as density increases, the hot outer cloud layers increasingly contribute to the emission, and therefore the line temperatures slowly rise with J-level.

On the other hand, isotopic CO line ratios for a specific transition are significantly determined by the optical depth of the cloud, i.e. both the gas column and volume density; fortunately, such line ratios do not suffer from unknown source sizes (beam filling factors) because of almost equal beam sizes of the compared lines.

The PDR model is able to reproduce the ${ }^{12} \mathrm{C}-{ }^{13} \mathrm{C}$ fractionation in molecules at intermediate gas densities via a charge-exchange reaction (for example, ${ }^{13} \mathrm{C}^{+}+{ }^{12} \mathrm{CO} \rightleftharpoons$ $\left.{ }^{12} \mathrm{C}^{+}+{ }^{13} \mathrm{CO}+36 \mathrm{~K}\right)$ : with an input of a ${ }^{12} \mathrm{C} /{ }^{13} \mathrm{C}$ abundance ratio of 66 we obtain a column density ratio of $N_{12 \mathrm{CO}} / N_{13 \mathrm{CO}}=33$; Henkel et al. (1998, and references therein) in fact derive a value of the ${ }^{12} \mathrm{C} /{ }^{13} \mathrm{C}$ ratio in molecules of 30 to 40 for IC 342 .

A PDR model appears also to be justified by the observation of strong $158 \mu \mathrm{m}$ [C II] fine structure line emission (Stacey et al. 1991). The interstellar UV radiation field in the nuclear region of IC 342 can be determined within a factor of order 2 to be $\chi=10^{3} \chi_{0}$ (see Fig. 18 of Stacey et al.) Much higher values of $\chi$ are found for sites of massive star formation like Orion A (see Stacey et al. 1993) and are unrealistic as an average over such large areas of 
Table 2. CO line ratios \& derived parameters

\begin{tabular}{|c|c|c|c|c|c|c|c|c|}
\hline cloud & offset & $R_{12} \frac{(3-2)^{1)}}{(2-1)}$ & $R_{13} \frac{(3-2)^{1)}}{(2-1)}$ & $R_{12 / 13}(3-2)^{2)}$ & $R_{12 / 13}(2-1)$ & $n\left(\mathrm{H}_{2}\right)$ & size & $M_{\mathrm{H}_{2}}$ \\
\hline & " & & & & & $10^{4} \mathrm{~cm}^{-3}$ & $\mathrm{pc}$ & $10^{6} M_{\odot}$ \\
\hline G & $(4,12)$ & 0.80 & 0.75 & $10.5(18.0)$ & 8.5 & 1.5 & 25 & 1. \\
\hline $\mathrm{D}$ & $(4,8)$ & 0.80 & 0.75 & $8.0(12.0)$ & 7.5 & 1.5 & $<25$ & $<2$. \\
\hline $\mathrm{C}$ & $(4,4)$ & 0.85 & 0.77 & $7.5(8.0)$ & 6.5 & 2 . & 35 & 4. \\
\hline $\mathrm{F}$ & $(0,-4)$ & 0.65 & 0.65 & $6.5(7.0)$ & 7.5 & 1 & 25 & 2 . \\
\hline A & $(4,-4)$ & 0.70 & 0.60 & $9.0(16.0)$ & 7.5 & 1 & - & - \\
\hline B & $(0,0)$ & 0.45 & 0.55 & 8.0 & 9.5 & 0.5 & - & - \\
\hline $\mathrm{E}$ & $(-4,-4)$ & 0.50 & 0.55 & $8.0(10.5)$ & 8.0 & 0.5 & - & - \\
\hline $\mathrm{H}$ & $(-4,-8)$ & 0.60 & 0.50 & $8.5(15.5)$ & 7.0 & $<0.5$ & $<20$ & $<0.6$ \\
\hline
\end{tabular}

1) Ratio of (3-2) to (2-1) line peak intensities for ${ }^{12} \mathrm{CO}$ and ${ }^{13} \mathrm{CO}$, resp., smoothed to $12^{\prime \prime}$ beam size $\left({ }^{12} \mathrm{CO}(2-1)\right.$ data by Eckart et al. 1980; errors are within 15\%).

2) Ratio of ${ }^{12} \mathrm{CO}$ and ${ }^{13} \mathrm{CO}$ intensities smoothed to $12^{\prime \prime}$ beam size ( $8^{\prime \prime}$ values in brackets, ${ }^{12} \mathrm{CO}(2-1)$ data by Eckart et al. 1980).

order 50 pc (equivalent to our spatial resolution for $\mathrm{CO}(3-$ $2)$ ); lower $\chi$ values are unlikely because of the observed dust temperature of order $40 \mathrm{~K}$ (Becklin et al. 1980) over an area of $60^{\prime \prime}$. Büttgenbach et al. (1992) obtain the same value of $10^{3} \chi_{0}$ modelling their C I observations.

Adopting element abundances of the gas phase to be entirely solar, one particular problem we are faced with in this PDR model is that ${ }^{13} \mathrm{CO}$ becomes optically thick on very short path lengths which results in a rather unlikely small range of gas column densities fitting the various observed ${ }^{12} \mathrm{CO} /{ }^{13} \mathrm{CO}$ line ratios. One possibility to overcome this problem is to assume element abundances (compared to hydrogen) not to be fully solar; if we reduce the element abundances of carbon and oxygen to $X[\mathrm{C}]=110^{-4}$ and $X[\mathrm{O}]=210^{-4}$ relative to $\mathrm{H}_{2}$, the range of column densities reaches reasonable values. Downes et al. (1992) give a value of $X[\mathrm{CO}]=10^{-5}$ to $510^{-5}$. Another possibility of "fine tuning" of the model would be to increase the strength of the UV radiation field by a factor of order 2. Nevertheless, we emphasize that this affects only the resulting column densities but not the gas density.

Specific results for several observed positions which fit the observed $\mathrm{CO}(3-2)$ and $(2-1)$ line ratios are given in Table 2. Figure 7 shows the excitation plot for cloud C. The average gas density for the clouds must be in the range of 0.5 to $210^{4} \mathrm{~cm}^{-3}$, i.e. slightly below the critical density for the $\mathrm{CO}(3-2)$ line which in all cases appears to be not fully thermally excited. Lower densities are unlikely because $(3-2) /(2-1)$ line ratios as also the absolute ${ }^{13} \mathrm{CO}(3-2)$ line intensities would drop below the observed values; higher densities would imply ${ }^{12} \mathrm{CO}$ and ${ }^{13} \mathrm{CO}\left(3_{-}^{-}\right.$ $2) /(2-1)$ ratios higher than observed. For cloud $\mathrm{C}$ we derive from its column density $\left(210^{23} \mathrm{~cm}^{-2}\right)$ and its linear extent a mass of $410^{6} M_{\odot}$ which is comparable to Sgr B2. Ratios of observed line temperatures to modelled brightness temperature values yield area filling factors of order 0.2 for the cloud positions which would be reduced to 0.1 if one attributes half the emission to the denser clouds and the other half to the underlying pedestal of intercloud gas. Because of the uncertainties of element abundances, gas column densities are more difficult to confine. However, the derived column densities agree with values by Eckart et al. (1990), Turner \& Hurt (1992) and Güsten et al. (1993). For the pedestal zone of emission (offset from the clouds) we obtain slightly lower values of the $(3-2) /(2-$ 1) line ratios but significantly higher isotopic line ratios which indicates that the gas has mainly lower column densities.

Regarding even higher excited $\mathrm{CO}$ emission we find that the $(4-3) /(2-1)$ line ratio observed for cloud $\mathrm{C}$ $(\mathrm{CO}(4-3)$ by Güsten et al. 1993) is well represented by our modelling, as well as the observed $(6-5) /(2-1)$ ratio (Harris et al. 1991) when the data are scaled to a $12^{\prime \prime}$ beam. Nevertheless, density gradients should be expected since a fixed gas density throughout a cloud of $\sim 30$ pc size can only be a first-order assumption; such gradients are in fact indicated by our HCN observations (Sect. 4.3).

\section{2. $\mathrm{CO}-[\mathrm{CII}]$}

Large-scale $[\mathrm{CII}]$ emission necessarily implies the existence of a PDR (Stacey et al. 1991). An important test to justify the PDR model for the CO observations is whether we succeed in modelling the $\mathrm{CO}$ and [C II] line observations at the same time. At present, such an investigation comparing emitting source sizes and beam filling factors must be preliminary because of the low angular resolution of the [C II] $158 \mu \mathrm{m}$ line observations.

Our model gives a $\mathrm{CO}(3-2)$ line brightness temperature of $18 \mathrm{~K}$ whereas $2 \mathrm{~K}$ is half the observed value (attributing half of the emission to the clouds themselves and the other half to the underlying pedestal in our $8^{\prime \prime}$ beam, which appears to be justified as a first guess facing the observed pedestal emission and a filling factor $\eta_{\mathrm{ff}}$ 


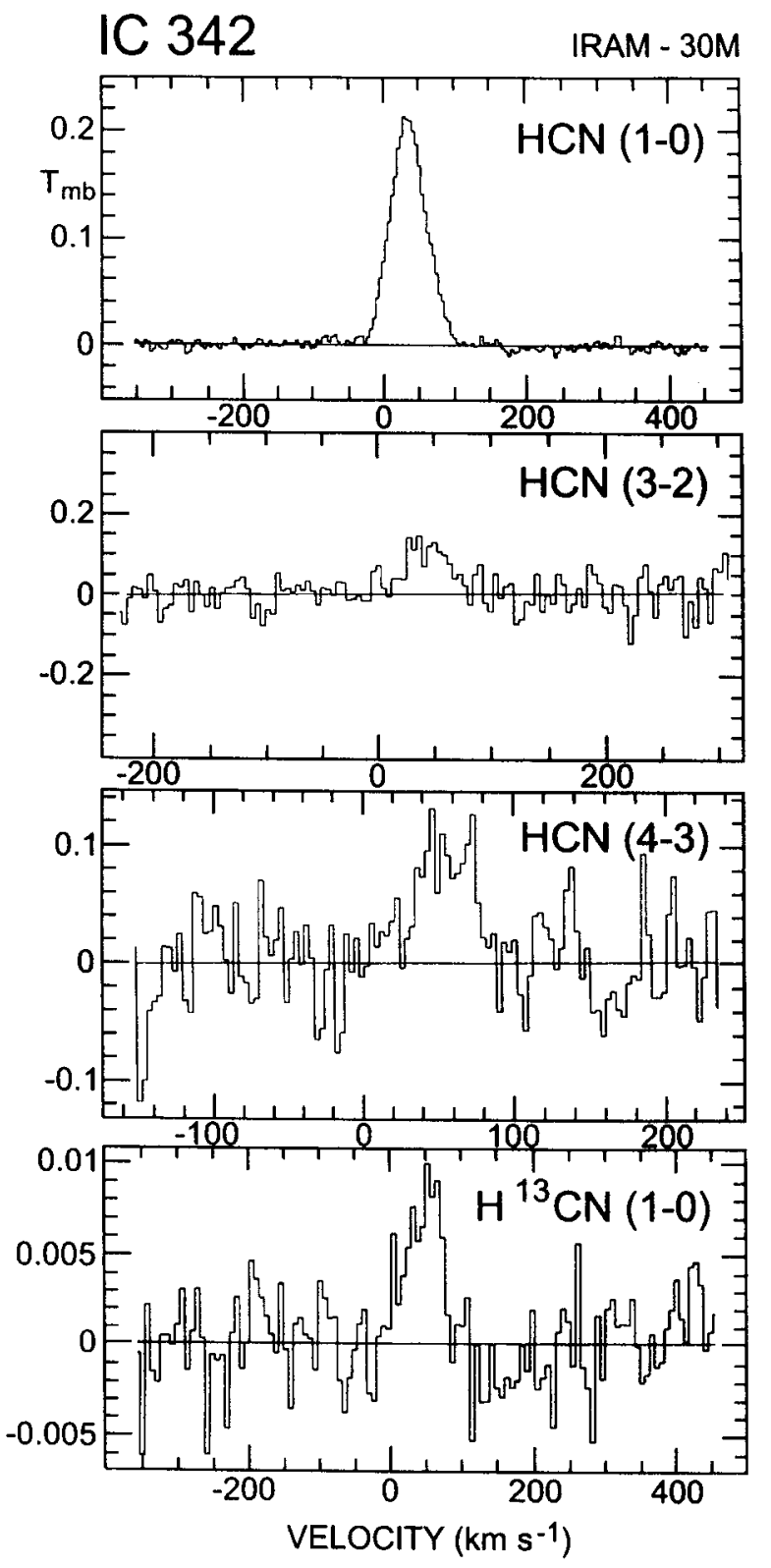

Fig. 5. HCN spectra observed at position of cloud C (offset $(4,4))$

considerably below 1, in general accordance with Stacey et al. 1993); hence, $\eta_{\mathrm{ff}}(\mathrm{CO})=0.13$ and the corresponding source size would be $3.5^{\prime \prime}$. Our modelled [C II] line brightness temperature is $25 \mathrm{~K}$, and $0.5 \mathrm{~K}$ is half of the observed value within the $55^{\prime \prime}$ beam; hence, $\eta_{\mathrm{ff}}([\mathrm{C} \mathrm{II}])=0.02$ which results in fact in the same $3.5^{\prime \prime}$ source size if we adopt to find the equivalent emission of five identical cloud sources within the large beam. Therefore, CO and [C II] lines may in fact be emitted from the same regions.

\subsection{The HCN measurements}

To explain the HCN line observations, we are not able at present to use the PDR model since $\mathrm{HCN}$ is not yet included in the chemical network. Therefore, we have to restrict our modelling to a "classical" LVG photon escape

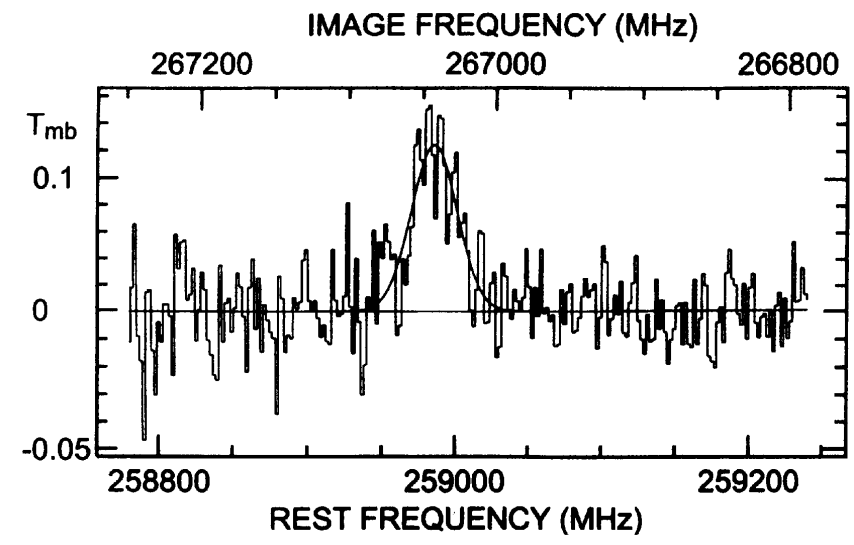

Fig. 6. " $\mathrm{H}^{13} \mathrm{CN}(3-2)$ " spectrum at the position of cloud C; the identification as a pair of ethanol $\left(9_{8}-8_{8}\right)$ transitions at a rest frequency of $267.045 \mathrm{GHz}$ is still very questionable

a)

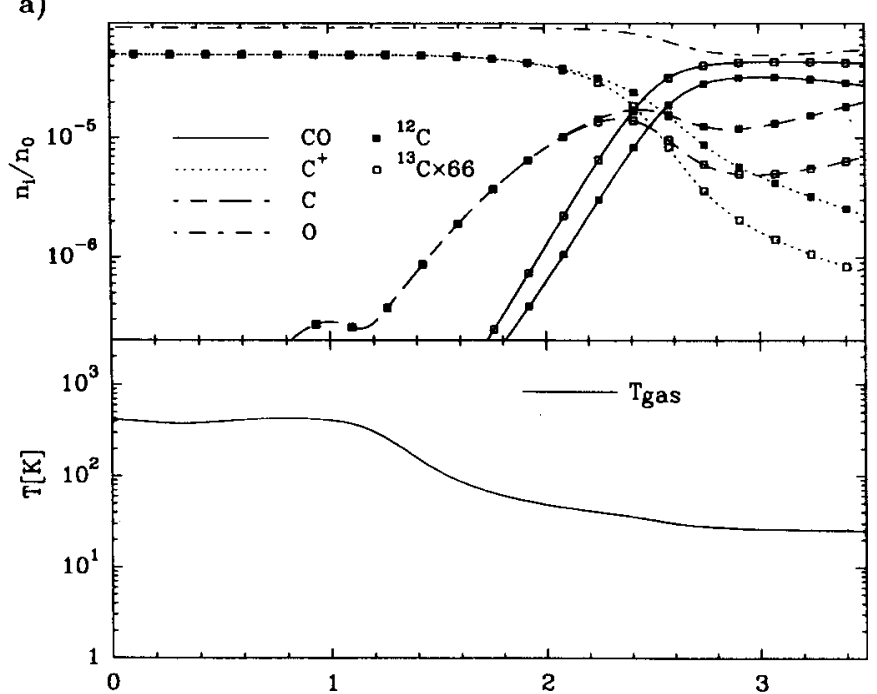

b)

Av

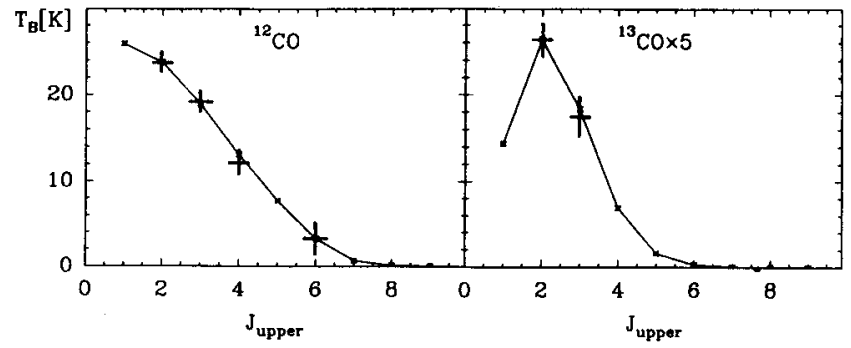

Fig. 7. Excitation plot of ${ }^{12} \mathrm{CO}$ line emission for cloud $\mathrm{C}$ using PDR model results given in Table 2; a) relative atomic/molecular abundances and temperature structure; b) brightness temperature $T_{\mathrm{b}}$ versus upper transition level (similar to Figs. 2 and 8a in Köster et al. 1994). Crosses denote observed line temperatures (scaled to a $12^{\prime \prime}$ beam) with reference to the $(2-1)$ transitions

probability method. We expect that this should give not too unrealistic results because $\mathrm{HCN}$ lines are excited in clumps in the inner parts of clouds, at 10 to 100 times higher gas densities than CO, where typical PDR effects much less dominate the molecular chemistry than in those outer clump layers where much of the $\mathrm{CO}$ emission is 
produced. Disadvantageously, we are not able to account for gradients in density and temperature.

Keeping the $\mathrm{H}^{12} \mathrm{CN} / \mathrm{H}^{13} \mathrm{CN}$ ratio to be 40 and varying the kinetic temperature, gas density, and gas column density as free parameters, we obtain sets of model clouds with HCN line brightness temperature ratios. To compare those with the observed line ratios, we first have to scale the line intensities (all observed with the same telescope) all to the same beam size (see Table 3). To do this we adopt for cloud $\mathrm{C}$ the size determined by Downes et al. (1992) from their interferometric HCN (1-0) map; we take into account that large beams could include more than one cloud source. Since this source size is expected to be an upper limit for the higher transitions, our excitation analysis yields a lower limit to the gas density. For clouds F, G and $\mathrm{H}$ we had to make assumptions from CO sizes (Table 1). Our derived column density is mainly determined by the $\mathrm{H}^{12} \mathrm{CN} / \mathrm{H}^{13} \mathrm{CN}$ ratio of the (1-0) transition, whereas our observed limit of $\mathrm{H}^{13} \mathrm{CN}(3-2)$ is not sensitive enough to give useful constraints.

We now discuss the conditions in more detail for cloud $\mathrm{C}$ because this is the most prominent cloud unambigously observed in all molecular transitions. With our five observed HCN transitions we obtain a rather definite modelling solution; in particular, the combination of $\mathrm{HCN}(4-3)$ and $\mathrm{H}^{13} \mathrm{CN}(1-0)$ very sensitively determines the resulting parameters (under the assumption that no temperature and density gradients are present). An excitation plot is given in Fig. 8.

As an additional important check, our modelling solution yields not only sensible line ratios but also sensible absolute line brightness temperatures as expected when considering filling factors from beam and source sizes. The best fitting solution yields $T_{\mathrm{k}}=30 \mathrm{~K}, n\left(\mathrm{H}_{2}\right)=10^{6} \mathrm{~cm}^{-3}$ and $N_{\mathrm{HCN}}=10^{13} \mathrm{~cm}^{-2}$. Nevertheless, the parameter set $\left(20 \mathrm{~K}, 210^{6} \mathrm{~cm}^{-3}, 10^{13} \mathrm{~cm}^{-2}\right)$ still gives a fair fit to the observations (see Fig. 8, open circles). Higher kinetic temperatures are unlikely because this would raise the HCN (3$2) /(1-0)$ ratio beyond the observed value or, alternatively, would decrease the (4-3) as well as the isotopic (1-0) line intensities too much due to lower $\tau$ values for both. Higher gas densities are unrealistic because under the assumption of source size of Table 1 the (3-2) line is subthermally excited, lower densities would yield a too low (4-3) intensity. We again emphasize the high importance of $\mathrm{HCN}(4-3)$ observations, because with these only very subthermally excited spectra we are able to constrain the gas density quite well. The column density is confined by the isotopic (1-0) line ratio and in accordance with all other line ratios.

With an HCN abundance ratio relative to hydrogen of $210^{-8}$ (Downes et al. 1992) we obtain a total hydrogen column density of $210^{22} \mathrm{~cm}^{-2}$ resulting in a mass of $210^{5} M_{\odot}$ with the adopted clump size. This high-density gas mass is of order $5 \%$ of the mass derived from the $\mathrm{CO}$ line observations, the $\mathrm{CO}$ emitting gas being at a density of $210^{4} \mathrm{~cm}^{-3}$. Such a mass fraction is consistent with the difference of beam filling factors for $\mathrm{CO}$ and $\mathrm{HCN}$ emission. If we, on the other hand, calculate the HCN emitting

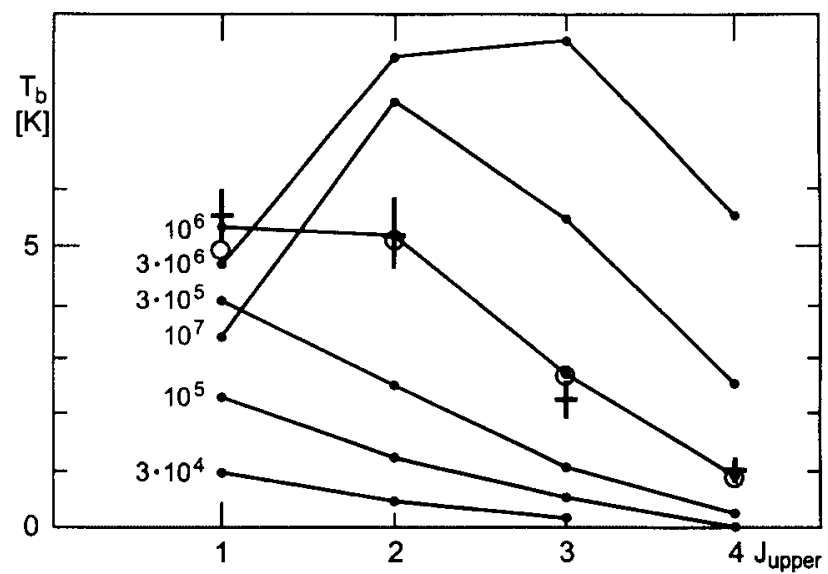

Fig. 8. Excitation plot of HCN line emission (modelled line brightness temperature $T_{\mathrm{b}}$ versus upper transition level) for cloud C using LVG model results with $T_{\mathrm{k}}=30 \mathrm{~K}$ and $N_{\mathrm{HCN}}=$ $10^{13} \mathrm{~cm}^{-2}$, and $n\left(\mathrm{H}_{2}\right)$ as indicated (open circles: $T_{\mathrm{k}}=20 \mathrm{~K}$, $\left.N_{\mathrm{HCN}}=10^{13} \mathrm{~cm}^{-2}, n\left(\mathrm{H}_{2}\right)=210^{6} \mathrm{~cm}^{-3}\right)$. Observed values multiplied by 17 are marked by crosses

gas mass by multiplying the gas density of $10^{6} \mathrm{~cm}^{-3}$ by the volume of the HCN clump, we obtain a mass which is orders of magnitude higher than the previous result; this means that the very dense gas must be highly clumped (low volume filling factor).

For the other cloud positions (F, G, H) we find modelling results given in Table 3 which are less accurate due to uncertain source sizes.

\section{Discussion}

\subsection{Intensity distribution/cloud morphology}

\subsubsection{CO maps}

To compare ours with other molecular line maps we predominantly consider those with angular resolutions of $\sim 10^{\prime \prime}$ or better. CO has been mapped with resolutions $\leq 10^{\prime \prime}$ only in the lowest transitions. A close inspection of such maps of ${ }^{12} \mathrm{CO}(1-0)$ (Ishizuki et al. 1990, 2.4" res., Wright et al. $1993,4.3^{\prime \prime} \times 2.5^{\prime \prime}$ res.; Meier et al. 2000, $4.5^{\prime \prime}$ res.) and ${ }^{13} \mathrm{CO}(1-0)$ (Turner \& Hurt 1992, 4.9" $\times 4.1^{\prime \prime}$ res., Wright et al. $1993,4.3^{\prime \prime} \times 2.5^{\prime \prime}$ res.; Meier et al. 2000, 4.5" res.) emission confirmes the general morphology concerning the mini-spiral, but there are interesting differences in the details. The exact positions of the emission maxima are slightly (but in some cases possibly not significantly) different for all the lines. Almost coincident emission maxima are observed in all $\mathrm{CO}$ maps (including ours) for clouds $\mathrm{C}, \mathrm{G}$ and $\mathrm{H}$; also their line centre velocities agree. This holds also for cloud D which in all these maps is not very prominent along the northern ridge of the mini-spiral. 
Cloud $\mathrm{E}$ is almost blended with emission from cloud $\mathrm{B}$ in the two ${ }^{12} \mathrm{CO}(1-0)$ maps, whereas in all three ${ }^{13} \mathrm{CO}(1-$ 0 ) maps E prominently appears but cloud B does not. However, in both our isotopic $\mathrm{CO}(3-2)$ maps cloud E along the ridge of the mini-spiral can be supposed in some of our channel maps; but at the position of B the emission falls off rather steeply and B is even not visible in any of our channel maps.

Cloud A is very clearly visible in all CO (1-0) maps of $\leq 5^{\prime \prime}$ resolution, but it can be identified only in our $10 \mathrm{~km} \mathrm{~s}^{-1}$ wide channel maps at 45 and $55 \mathrm{~km} \mathrm{~s}^{-1}$ centre velocity; this is also the case in the $7^{\prime \prime}{ }^{12} \mathrm{CO}(1-0)$ map of Lo et al. (1984).

\subsubsection{Other molecular line and continuum maps}

Including also the HCN (1-0) map of Downes et al. (1992, $2.7^{\prime \prime}$ res.) and the $\mathrm{NH}_{3}$ map of Ho et al. (1990, $5^{\prime \prime}$ res.) in our discussion we again see reasonable agreement in the morphological and kinematical structure for clouds C, D, $\mathrm{G}, \mathrm{E}$ and $\mathrm{H}$, but differences for clouds $\mathrm{A}$ and $\mathrm{B}$ :

Cloud A is very prominent in the $\mathrm{HCN}(1-0)$ and lowest CO transitions, but rather weak in both isotopes of $\mathrm{CO}(3-2)$ emission (also, $\mathrm{HCO}^{+}$emission is weak here, see Q-Rieu et al. 1992) and absolutely absent in $\mathrm{NH}_{3}$; furthermore, the centre velocity of $\mathrm{HCN}(1-0)$ and of our CO spectra differ by $>10 \mathrm{~km} \mathrm{~s}^{-1}$. One possible explanation for the absence of $\mathrm{NH}_{3}$ and prominent $\mathrm{CO}(3-2)$ emission is that this cloud is very cold.

The case for cloud B is even more confusing; it is coincidently seen in ${ }^{12} \mathrm{CO}(1-0)$ and in $\mathrm{HCN}(1-0)$; this is surprising because both lines should trace gas at different volume densities, and $\mathrm{B}$ does not appear in ${ }^{13} \mathrm{CO}(1-0)$ nor in both $\mathrm{CO}(3-2)$ transitions. The lack of ${ }^{13} \mathrm{CO}$ emission would imply low optical depth, i.e. low column density, the high HCN emission would mean high volume density; both arguments would point at gas with an extremely low volume filling factor.

Inspecting maps of free-free (ff)-emission from H II regions at $6 \mathrm{~cm}$ by Becklin et al. (1980, $2^{\prime \prime}$ resol.) and Turner \& Ho (1983, 1" resol.), some of the emission maxima appear to be closely associated with clouds A through $\mathrm{H}$. The strongly clumped $\mathrm{H}_{\alpha}$ emission $\left(\mathrm{H}_{\alpha}\right.$ image presented in Meier et al. 2000) does mostly not coincide with ffemission maxima, naturally explained by extinction of clumpy dust; we expectedly find $\mathrm{H}_{\alpha}$ also anti-coinciding with molecular line emission, but it is also not concentrated to cloud B.

Although the very details are difficult to judge, it looks as if a number of HII regions (ff-emission clouds) are surrounding the centre of IC 342 like a chain of pearls which are associated with the molecular clouds situated in the central mini-spiral, the largest of those we observe as clouds A, B, C, E. Hence, not only cloud B - as stated by Turner \& Hurt (1992) and several other investigations - but all of them near the centre of IC 342 show moderate star formation activity.

\subsection{The different components of the gas}

Our CO analysis reveals several clouds of $\leq 20$ to about $40 \mathrm{pc}$ deconvolved sizes with masses of $\leq 410^{6} M_{\odot}$ distributed around the centre of IC 342 and along the minispiral. The central clouds are associated with moderate ffemission indicating star formation activity similar to our Galactic Centre.

The gas of the clouds observed in ${ }^{12} \mathrm{CO}$ has high optical depths $\left(\tau_{12} \sim 10\right)$ while ${ }^{13} \mathrm{CO}$ is moderately optically thick $\left(\tau_{13} \sim 0.4\right)$. $(3-2) /(2-1)$ CO line ratios are very similar for both ${ }^{12} \mathrm{CO}$ and ${ }^{13} \mathrm{CO}$ and therefore indicate that both ${ }^{12} \mathrm{CO}$ and ${ }^{13} \mathrm{CO}$ lines are emitted from gas at similar average density, i.e. not from completely different regions. Also, our ${ }^{13} \mathrm{CO}$ maps show the same morphology as ${ }^{12} \mathrm{CO}$ for the central area, we derive similar beam filling factors for both isotopes, and the line profiles are identical; that means that the gas components emitting ${ }^{12} \mathrm{CO}$ and ${ }^{13} \mathrm{CO}$ seem to be well mixed at the positions of the clouds, although the main parts of the ${ }^{12} \mathrm{CO}$ and ${ }^{13} \mathrm{CO}$ emission flux stem from different depths of the clouds.

Hence, for the dense clouds we see no evidence to imply an additional gas component being observed in ${ }^{12} \mathrm{CO}$ but not in ${ }^{13} \mathrm{CO}$, as Wall \& Jaffe (1990) do. We find, on the other hand, no evidence for any optically thin ${ }^{12} \mathrm{CO}$ emission (see Wall \& Jaffe 1990). ${ }^{12} \mathrm{CO}$ emission appears to originate not only from extended ridges but also from the dense clouds. Furthermore, single-dish as well as interferometer observations should not trace completely different gas components in ${ }^{12} \mathrm{CO}$ and ${ }^{13} \mathrm{CO}$ : although ${ }^{12} \mathrm{CO} /{ }^{13} \mathrm{CO}$ ratios as low as 10 or even lower might be blends of drastically different regions, such low ratios are found by single dish measurements as well as by interferometer measurements (Wright et al. 1993) down to a few arcsec resolution; that means that only at much higher resolution one should be able to observe the clouds substructure, i.e. to distinguish sharply between very dense clumps and interclump gas - or one has to use different tracers (see below).

Our results are in good agreement with column densities and masses found by Güsten et al. (1993) $\left(N_{\mathrm{H}_{2}}=\right.$ $310^{23} \mathrm{~cm}^{-2}$ and $M_{\mathrm{H}_{2}}=4.210^{6} M_{\odot}$ for the central $\left.15^{\prime \prime}\right)$, and Downes et al. (1992) $\left(\sim 10^{6} M_{\odot}\right.$ for cloud C) and Wright et al. (1993) (clouds of $\leq 20$ to 50 pc with $\sim 10^{6} M_{\odot}$ each). Turner \& Hurt (1992) derive a slightly higher mass of $910^{6} M_{\odot}$ for the central $14^{\prime \prime}(120 \mathrm{pc})$.

On larger scales, the intercloud gas contributes an increasing fraction to the total gas mass $\left(410^{7} M_{\odot}\right.$ within $23^{\prime \prime}$, Wall \& Jaffe $1990,4.210^{7} M_{\odot}$ within $56^{\prime \prime}$, Turner \& Hurt 1992, $210^{8} M_{\odot}$ for the total central region, Eckart et al. 1990); this is a factor of order 10 lower than the dynamical mass of the centre of IC $342\left(1.210^{9} M_{\odot}\right.$ within 1500 pc, Young \& Scoville 1982) and would imply a "normal" mass ratio of interstellar matter compared to stars.

Although the PDR model intrinsically considers gradients in temperature as the gas temperature is calculated locally, it operates with the input of a fixed gas density. Our 1-component model with a fixed $\mathrm{H}_{2}$ density (2 $10^{4} \mathrm{~cm}^{-3}$ for cloud C) reproduces our observed line 
Table 3. Observed HCN line peak intensities ${ }^{1)} \&$ model results

\begin{tabular}{|c|c|c|c|c|c|c|}
\hline line & $\begin{array}{c}\text { cloud } \\
\text { off pos. }\end{array}$ & $\begin{array}{c}\mathrm{C} \\
(4,4)\end{array}$ & $\begin{array}{c}\mathrm{F} \\
(0,-4)\end{array}$ & $\begin{array}{c}\mathrm{G} \\
(4,12)\end{array}$ & $\begin{array}{c}\mathrm{H} \\
(-4,-8)\end{array}$ & \\
\hline $\mathrm{HCN}(1-0)$ & & 0.33 & 0.33 & 0.30 & 0.30 & (in Kelvin) \\
\hline $\mathrm{H}^{13} \mathrm{CN}(1-0)$ & & $0.01(4)$ & $0.01(4)$ & $\leq 0.01$ & $\leq 0.01$ & \\
\hline $\operatorname{HCN}(2-1)$ & & 0.30 & 0.25 & - & - & \\
\hline $\mathrm{HCN}(3-2)$ & & 0.13 & 0.11 & $0.08(4)$ & $0.04(7)$ & \\
\hline $\mathrm{HCN}(4-3)$ & & 0.06 & $0.04(5)$ & $0.03(4)$ & $\leq 0.01(5)$ & \\
\hline \multicolumn{7}{|l|}{ parameters } \\
\hline$n\left(\mathrm{H}_{2}\right)$ & & 1. & 0.6 & 0.3 & $\leq 0.1$ & $10^{6} \mathrm{~cm}^{-3}$ \\
\hline$M_{\mathrm{H}_{2}}$ & & 0.2 & $(0.3)$ & $(0.1)$ & $(0.07)$ & $10^{6} M_{\odot}{ }^{2)}$ \\
\hline
\end{tabular}

1) Scaled to a $10^{\prime \prime}$ beam size (from source sizes of Table 1 and measured beam sizes).

2) Source sizes from Table 1.

ratios for the cloud positions even including the $\mathrm{CO}(4-3)$ line by Güsten et al. (1993) as well as the CO (6-5) line by Harris et al. (1992). On the other hand, Güsten et al. also state that most of the total column density and mass is contributed by their denser model cloud component.

The clouds themselves must have a clumpy substructure (low volume filling factor). This becomes obvious because masses calculated from average densities and source sizes largely exceed the values derived from column densities. The clumpiness should allow the interstellar radiation field to penetrate the clouds heating the clumps "from outside". This idea is in accordance with the [C II] emission reported in Stacey et al. (1991); Hollenbach \& McKee (1989) point out that the [C II] emission should be due to ionization by UV radiation and should not be produced by shocks. Their finding that C II generally is associated with $\mathrm{CO}$ emission agrees with our result that C II and $\mathrm{CO}$ are likely to be emitted from the same regions (see Sect. 4.2). In fact, we can completely explain the $\mathrm{CO}$ and [C II] observations with a PDR model which was queried previously (e.g. Wall \& Jaffe 1990). As an important consequence, this scenario implies that denser parts of the clouds should be cooler than the less dense outer layers (in contradiction to LVG model results, see Sect. 4.1.1).

Our HCN analysis nicely confirms this model: There exists a dense gas component $\left(n\left(\mathrm{H}_{2}\right)=10^{6} \mathrm{~cm}^{-3}\right.$, see Sect. 4.3) contributing about $5 \%$ to the total cloud mass (in rough accordance with Paglione et al. 1997), and this component again is clumpy and has a temperature of mostly $30 \mathrm{~K}$; that is just the temperature we obtain for the interior of our PDR cloud model. A further hint at cool temperatures for the denser components is given by Eckart et al. (1990) who remark that at a temperature for the bulk of the dust of $42 \mathrm{~K}$ the derived dust mass falls short by a factor of 5 , but this is compensated if one adopts $30 \mathrm{~K}$ instead. We find no evidence for a dense component which is as warm as $50 \mathrm{~K}$ or more. We think that the older findings of a warm dense component are an artefact of the LVG models which are unable to consider temperature gradients in the calculations. On the other hand, one seems to observe gradients in temperature and density everywhere on all scales throughout the interstellar medium, in our and in external galaxies, in particular when observing large areas within the beam. Such gradients can change the optical depths of molecular lines considerably compared to constant LVG conditions, and this effect might be particularly large for the outer layers of clouds when they are exposed to soft UV radiation which is the case in the centre of IC 342 by Stacey et al. (1991).

Temperature gradients may also play an important role for the $\mathrm{NH}_{3}$ emission (Ho et al. 1990) revealing temperatures of 70 to $100 \mathrm{~K}$ : the observed lines are optically thin, and therefore the beam penetrates all interstellar matter within it; it might stem from warm gas of intermediate densities, either an extended component or the outer layers of the clouds, and not from the dense regions of the small cores. The total mass of the warm gas seen in $\mathrm{NH}_{3}$ of only $10^{6} M_{\odot}$ (Ho et al. 1990) could support this idea.

However, temperature gradients should be much less important for the innermost cloud cores which could be shielded from UV in case they are dense enough (see Köster et al. 1994; van Dishoeck \& Black 1998). Hence, we expect the results of our HCN analysis - which does not take gradients into account - to deviate not largely from real conditions in case the dense cores in our beam do not contain interior strong UV sources. Our HCN analysis is also in accordance with Serabyn \& Güsten (1991) who obtain an upper limit of the density of the dense gas component of $510^{5} \mathrm{~cm}^{-3}$ from an upper limit of $10 \mathrm{mK}$ for the CS (7-6) line emission adopting $T_{\mathrm{k}}=70 \mathrm{~K}$; this density limit would rise by at least a factor of 2 when adopting less than half the temperature.

Apart from the dense clouds, we observe a pedestal of more extended CO emission. Interestingly, (3-2)/(21) line ratios of ${ }^{12} \mathrm{CO}$ do not drop off as expected for much lower gas densities (like Wright et al. 1993, obtain); 
instead, ${ }^{13} \mathrm{CO}$ emission is considerably lower hinting at decreasing gas column densities. It is difficult to check this result by observations with much higher angular resolution than ours because interferometers lose much of the flux from extended components.

\subsection{The nucleus of IC 342 and the Galactic Centre}

Poglitsch et al. (1991, and references therein) report a $158 \mu \mathrm{m}$ [C II] line luminosity of $210^{3} L_{\odot}$ within an area of $30 \mathrm{pc}^{2}$ and of $310^{4} L_{\odot}$ within $860 \mathrm{pc}^{2}$ towards the Galactic Centre region. Extrapolating the first value from 30 to $860 \mathrm{pc}^{2}$ yields $5.710^{4} L_{\odot}$ which is surprisingly close to the observed value regarding the vast difference of the areas (only a factor of 2 larger than observed, easily attributed to an area filling factor $<1$ ). This shows that on large scales the $[\mathrm{CII}]$ emission is distributed rather evenly and wide-spread across the Galactic Centre region. This also seems to be the case for the UV-excited $2.1 \mu \mathrm{m} \mathrm{H}_{2}$ emission (Pak et al. 1996). The remarkable correlation between molecular clouds and [C II] emission and, in particular, H II regions associated with such clouds and peaks of [C II] emission supports the conclusion that on large scales the massive Galactic Centre clouds are heated to a large fraction by the external (soft UV-) radiation field produced by a widely distributed population of B stars (Poglitsch et al. 1991).

On the other hand, most of the molecular gas at higher densities $\left(\geq 10^{4} \mathrm{~cm}^{-3}\right)$ appears to be cooler compared to the low-density gas $\left(T_{\mathrm{k}} \sim 100 \mathrm{~K}\right.$ at $\left.n\left(\mathrm{H}_{2}\right) \leq 10^{3} \mathrm{~cm}^{-3}\right)$ : an $\mathrm{SiO}$ line analysis at 33 clump positions within the inner $\pm 200 \mathrm{pc}$ of the Galactic Centre region (see Hüttemeister et al. 1998 and references therein) reveals evidence that this (dense) gas is now at $T_{\mathrm{k}} \sim 20-30 \mathrm{~K}$ only, although $\mathrm{SiO}$ is thought to be released from grains into the gas phase by supersonic collision processes (which heat the gas to high temperatures). This is in accordance with the idea that the high-temperature phase producing gaseous $\mathrm{SiO}$ has a short energy dissipation time scale (see Güsten 1989 and references therein), and at present these clouds are cool inside, their outer layers being heated to a large fraction from outside.

In comparison, in IC 342 we infact seem to find a very similar situation: a large fraction of the $[\mathrm{CII}]$ emission is likely to be emitted from the outer layers of the central molecular clouds observed in CO (see Sect. 4.2) which appear to be partly correlated with H II regions (Sect. 5.1.2). Also, the dense gas component observed in $\mathrm{HCN}(\leq 30 \mathrm{~K})$ is rather cool (Paglione et al. 1997 remark that the observed HCN (1-0) luminosity is about the same as that of the inner $1 \mathrm{kpc}$ of our Galaxy).

Extrapolating the [C II] line luminosity of the Galactic Centre $\left(310^{4} L_{\odot}\right)$ within $860 \mathrm{pc}^{2}$ - just as a crude check - to $185000 \mathrm{pc}^{2}$ (the $55^{\prime \prime}$ beam area of the [C II] data in IC 342) yields $6.410^{6} L_{\odot} \cdot 2.610^{6} L_{\odot}$ is observed, only a factor of $\sim 2$ lower whereas the difference of areas is a factor of $\sim 200$ ! Hence, the total [C II] line luminosity of the nucleus of IC 342 and the Galactic Centre appear to be about the same, and therefore also the strength of the interstellar UV radiation field should be comparable in both galactic nuclei. Even the Galactic Centre observations carried out by the COBE satellite with its large beam ( $7^{0}$ equivalent to about $900000 \mathrm{pc}^{2}$ at a distance of $8 \mathrm{kpc}$ ) roughly agree with these numbers (Fixsen et al. 1999).

For the clouds in the centre of IC 342 we are able to explain the heating of the gas throughout the field of $0.5 \mathrm{kpc}$ extent observed in CO entirely by the interstellar soft UV radiation field which even heats the interior of the clouds (at $n\left(\mathrm{H}_{2}\right)=210^{4} \mathrm{~cm}^{-3}$ ) to $\sim 25 \mathrm{~K}$. Alternative heating processes may contribute only to the densest component. But, despite the destorted velocity field (Turner \& Hurt 1992) indicating non-circular motion around the centre of IC 342, we see no evidence for turbulent friction as a dominant heating source for the clouds at this time, nor that enhanced starburst should play any important role.

There is striking evidence that the physical properties of the molecular clouds in the nucleus of IC 342 and our Galactic Centre region are very similar, and therefore also the ongoing physical processes.

\section{Conclusions}

From our multi-line analysis probing the different components of molecular gas in the nucleus of IC 342, we draw the following conclusions:

(1) Embedded within a pedestal of $\mathrm{CO}$ emission we observe several giant molecular clouds (such as Sgr B2 in our Galactic Centre) whose morphological structure is in general accordance with previous investigations, possibly except clouds A and B;

(2) From our data for the clouds, we see no evidence that ${ }^{12} \mathrm{CO}$ and ${ }^{13} \mathrm{CO}$ are emitted from different regions; there is also no evidence for optically thin ${ }^{12} \mathrm{CO}$ emission of the clouds. While an LVG model approach yields results apparently contradicting our observations, we are able to model our $\mathrm{CO}$ observations and complementing [C II] data entirely in terms of a PDR model. While such an approach was successfully applied for the typical starburst galaxy M 82, (see Mao et al. 2000), it is remarkable that such a model is also required for a "normal" quiescent spiral galaxy. We even suspect that a PDR scenario might be applicable not only to these galactic nuclear regions but also to very large parts of the interstellar medium of galactic spiral disks;

The bulk of the gas of the GMC's with sizes of $\leq 20$ to $\sim 40$ pc and masses of $\sim 10^{6} M_{\odot}$ has an average density of order $10^{4} \mathrm{~cm}^{-3}$; it is highly clumped;

(3) Embedded in these GMC's we find denser gas $\left(\sim 10^{6} \mathrm{~cm}^{-3}\right)$ of up to $5 \%$ of their mass which itself is again highly clumped and cool $\left(T_{\mathrm{k}}=20-30 \mathrm{~K}\right)$;

(4) There is convincing evidence from our observations in accordance with other investigations that the large density gradients are connected with large temperature gradients where the thin gas is rather warm and temperature 
decreases with increasing density, as should be expected in a PDR scenario. The highly clumpy structure allows the soft UV radiation to penetrate well through all parts of the region; [CII] should be emitted to a large fraction (i.e. $\geq 50 \%$ ) from the outer layers of the identified clouds which in some cases show also moderate thermal ff-emission from associated H II regions;

(5) While we see no need to invoke other heating sources for the gas seen in $[\mathrm{C} \mathrm{II}] / \mathrm{CO}$, this should in proncipal be considered for the dense gas phase seen in $\mathrm{HCN}$ (LVG models do not tell anything about heating mechanisms): cosmic ray heating is only sufficient if the cosmic ray flux is very much higher than in the Galactic disk. Turbulent friction of clouds can not be ruled out, although the short energy dissipation times might cause problems with this mechanism. On the other hand, our PDR model yields a gas temperature of $\sim 25 \mathrm{~K}$ for the interior of the clouds, as is determined for the dense cores from the $\mathrm{HCN}$ data;

(6) We report a line detection at $267.042 \mathrm{GHz}$ whose identification as a pair of ethanol $\left(9_{8}-8_{8}\right)$ transitions is still uncertain;

(7) A comparison with our own Galactic Centre shows increasing similarities of these two galactic nuclear regions concerning the line as well as FIR continuum emission of the clouds and hence their physical conditions. Therefore, their evolution should be similar and it appears worth while to investigate in future this apparent "mirror image" of our own Galactic Centre using probes of very different states of excitation at much higher spatial resolution.

Acknowledgements. We like to thank H. Rothermel, A. Karpov, H. Hein, S. Navarro, D. John for their excellent and engaged support of our sometimes very experimental project, particularly when observing in the submm range. $\mathrm{H}$. Störzer assisted with the modelling. F. Bertoldi, C. Henkel, R. Launhardt, K. Menten and J. Stutzki, contributed useful discussions and comments.

\section{References}

Becklin, E. E., Gatley, I., Matthews, K., et al. 1980, ApJ, 236, 441

Büttgenbach, T. H., Keene, J., Phillips, T. G., \& Walker, C. K. 1992, ApJ, 397, L15

van Dishoeck, E. F., \& Black, J. H. 1988, ApJ, 334, 771

Downes, D., Radford, S. J. E., Guilloteau, S., et al. 1992, A\&A, 262,424

Draine, B. T. 1978, ApJS., 36, 595

Eckart, A., Downes, D., Genzel, R., et al. 1990, ApJ, 348, 434

Fixsen, D. J., Bennett, C. L., \& Mather, J. C. 1999, ApJ, 526, 207

Güsten, R., Serabyn, E., Kasemann, C., et al. 1993, ApJ, 402, 537
Güsten, R. 1989, IAU Symp. 136, ed. M. Morris, 89

Harris, A. I., Hills, R. E., Stutzki, J., et al. 1991, ApJ, 382, L75

Henkel, C., Chin, Y.-N., Mauersberger, R., \& Whiteoak, J. B. 1998, A\&A, 329, 443

Hollenbach, D., \& McKee, C. F. 1989, ApJ, 342, 306

Ho, P. T. P., Turner, J. L., \& Martin, R. N. 1987, ApJ, 322, L67

Ho, P. T. P., Martin, R. N., Turner, J. L., \& Jackson, J. M. 1990, ApJ, 355, L19

Hüttemeister, S., Dahmen, G., Mauersberger, R., et al. 1998, A\&A, 334, 646

Ishizuki, S., Kawabe, R., Ishiguro, M., et al. 1990, Nature, 344, 224

Jackson, J. M., Paglione, T. A. D., Carlstrom, J. E., \& Q-Rieu, N. 1995, ApJ, 438, 695

Köster, B., Störzer, H., Stutzki, J., \& Sternberg, A. 1994, A\&A, 284,545

Lo, K. Y., Berge, G. L., Claussen, M. J., et al. 1984, ApJ, 282, L59

Mao, R. Q., Henkel, C., Schulz, A., et al. 2000, A\&A, 358, 433

Martin, R. N., \& Ho, P. T. P. 1986, ApJ, 308, L7

Mauersberger, R., \& Henkel, C. 1989, A\&A, 223, 79

McCall, M. L. 1989, AJ, 97, 1341

Meier, D. S., Turner, J. L., \& Hurt, R. L. 2000, ApJ, 531, 200

Millar, T. J., Macdonald, G. H., \& Habing, R. J. 1995, MNRAS, 273, 25

Nummelin, A., Bergmann, P., Hjalmarson, A., et al. 2000, ApJS, 128, 213

Paglione, T. A. D., Jackson, J. M., \& Ishizuki, S. 1997, ApJ, 484, 656

Pak, S., Jaffe, D. T., \& Keller, L. D. 1996, ApJ, 457, L43

Poglitsch, A., Stacey, G. J., Geis, N., et al. 1991, ApJ, 374, L33

Q-Rieu, N., Jackson, J. M., Henkel, C., Bach, T., \& Mauersberger, R. 1992, ApJ, 399, 521

Rothermel, H., \& Gundlach, K. H. 1994, Archiv f. Elektrotechnik 77, 61

Serabyn, E., \& Güsten, R. 1991, IAU Symp. 146, ed. F. Combes, \& F. Casoli, 306

Stacey, G. J., Jaffe, D. T., Geis, N., et al. 1993, ApJ, 404, 219

Stacey, G. J., Geis, N., Genzel, R., et al. 1991, ApJ, 373, 423

Steppe, H., Mauersberger, R., Schulz, A., \& Baars, J. W. M. 1990, A\&A, 233, 410

Störzer, H., Zielinsky, M., Stutzki, J., \& Sternberg, A. 2000, A\&A, 358, 682

Turner, J. L., \& Ho, P. T. P. 1983, ApJ, 268, L79

Turner, J. L., \& Hurt, R. L. 1992, ApJ, 384, 72

Wall, W. F., \& Jaffe, D. T. 1990, ApJ, 361, L45

Wild, W., Greve, A., Karpov, A., Hein, H., \& Lamb, J. 1994, The IRAM $355 \mathrm{GHz}$ Receiver at the $30 \mathrm{~m}$ Telescope (Tech. Report)

Wright, M. C. H., Sshizuki, S., Turner, J. L., Ho, P. T. P., \& Lo, K. Y. 1993, ApJ, 406, 470

Wolfire, M. G., Hollenbach, D., \& Tielens, A. G. G. M. 1989, ApJ, 344, 770

Young, J. S., \& Scoville, N. 1982, ApJ, 258, 467 